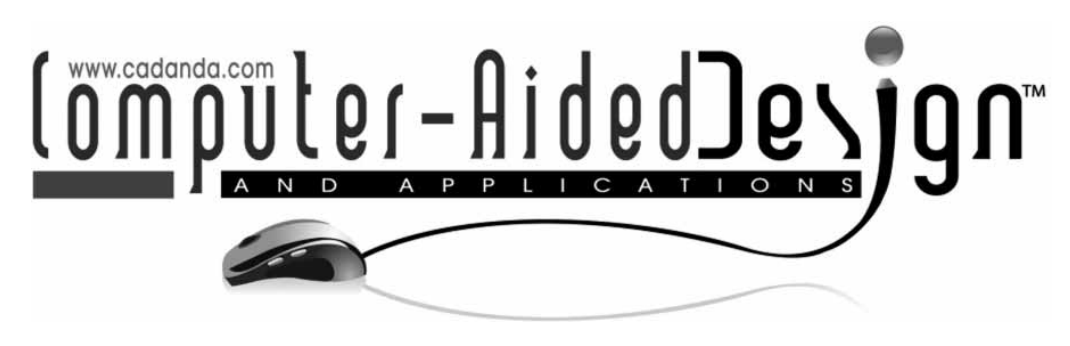

\title{
Slice Generation and Data Retrieval Algorithm for Rapid Manufacturing of Heterogeneous Objects
}

\author{
Vikas Gupta $^{1}$, VK Bajpai ${ }^{2}$ and Puneet Tandon ${ }^{3}$ \\ ${ }^{1}$ CDLMGEC, Sirsa, INDIA, vikasbrcm@rediffmail.com \\ ${ }^{2}$ NIT, Kurukshetra, INDIA \\ ${ }^{3}$ PDPM-IIITDM, Jabalpur, INDIA, ptandon@iiitdmj.ac.in
}

\begin{abstract}
With the development of Rapid manufacturing techniques, a heterogeneous object can be manufactured by processing geometric and material information in each layer while slicing the object. The functionality and surface finish of heterogeneous object depends upon precise slicing and accurate information processing i.e. geometric and material during fabrication. The current work focuses on both the issues. Layer thickness is varied using adaptive thickness method. Horizontal and vertical contours are approximated to reduce the error and minimize the effect of geometry and material stair-step effects. Reference based boundary mesh generation approach is used for defining material composition at each point in the slice domain. An algorithm is proposed for scanning layers to retrieve geometric and material information at each point in the object domain. A database system is developed for faster processing of information and to avoid the data redundancy during operation. Reference based approach, proposed algorithm and adaptive thickness method are implemented and some cases are discussed to show the validity of the work.
\end{abstract}

Keywords: rapid manufacturing, heterogeneous, slicing.

\section{INTRODUCTION}

Rapid manufacturing (RM) also called layered manufacturing (LM), produces parts layer by layer directly from 3D CAD models. An essential task in rapid manufacturing of homogeneous objects is the planning of fabrication, commonly referred as process planning tasks which includes orientation determination, support structure determination, slicing and deposition path planning. Slicing is one of the essential tasks in the 3D model layer domain. The layers are obtained by slicing the object, which can be thought of as a mapping from the 3D computer aided model to 2D layer.

Rapid manufacturing for homogeneous objects has been under development for many years and is now extended to fabrication of heterogeneous objects (HO). A variety of RM methods have been developed for manufacturing objects including Stereolithography, Laminated Object Manufacturing (LOM), Selective Laser Sintering (SLS), Fused Deposition Modeling (FDM), and Inkjet Printing. Some of these can manufacture heterogeneous objects. The quality of fabricated objects is based on their sliced data.
Different methods of slicing will result in different data for downstream applications. So, selection of slicing algorithm plays an important role in RP processing of heterogeneous objects. Different slicing algorithms are studied extensively in rapid manufacturing community to address various issues e.g. minimizing the error in contours, optimizing slice thickness, and improving surface finish etc. The issues related to error in slicing contours have been investigated e.g. [10,13]. Adaptive slicing method for reducing minimum fabrication time is developed [14]. This approach significantly reduces fabrication time by 17-37 percent compared to conventional methods and improved surface finish by eliminating unnecessary slices. However, there has been no examination of how the slicing issue is directly related to object tessellation besides a mention of the fact that over tessellation may be done. Tata et al. [13] discussed an adaptive slicing algorithm that can vary the slice thickness depending on the geometry of the object to be prototyped in an attempt to minimize layering error and improve surface finish. A direct slice CAD model using contours instead of going 
to Stereolithography (STL) is proposed, hence overcoming some problems that would be encountered [6]. Hope et al. [5] outlined the issues for improving geometric accuracy, while Choi et al. [1] dealt with a tolerant slicing algorithm to overcome computer memory constraints and computational problems. A review of various slicing methods for CAD and tessellated models are studied e.g. [10]. When an object is sliced, horizontal slice planes are intersected with the sides of the triangular facets at each particular slice height. Those collective intersecting points are then adjacently joined by straight lines to form contours at each height e.g. $[8,13]$. It is apparent that if there are more intersected triangles, there would be more points available, and thus, the contour would be more accurate. This process is repeated until contours are created for the entire object from top to bottom e.g. [8]. The slicing process will bring about a layering error that would affect the surface finish of the prototype e.g. [13]. The layering error increases with increased slice thickness e.g. [14]. In view of above discussion, an adaptive based slice generation procedure is used for rapid manufacturing of heterogeneous objects. Slicing errors are reduced to minimum for better surface finish.

RP techniques need point wise diverse data i.e. geometric and material, in the slice generated from 3D CAD model of heterogeneous objects. Reference based approach provides the internal structures and material distributions at every point in each region of the HO slice [3]. Optimizing the layer thickness and reducing the error is also a complex task. Further, it is a challenge to process and retrieve the geometric and material information efficiently at faster rate while slicing and rapid manufacturing of $\mathrm{HO}$.

In the present work, slicing process for rapid manufacturing of HO is classified into three categories; Pre-processing, Processing, Post processing which are described in Fig. 1.

Section 2 elaborates pre-processing tasks necessary for rapid manufacturing i.e. selecting the slicing plane, slicing direction and optimizing the layer thickness for geometric and material variation; Section 3 describes the processor module which slices the 3D CAD models into layers by generating contours, meshing and material mapping for downstream postprocessing tasks. Post processing task i.e. scanning each layer for processing geometric and material information is explained in Section 4; file format for storing retrieved data is discussed in Section 5; the proposed algorithms are implemented and verified by illustrating some examples and performance of database is analyzed in Section 6 and finally, the paper is concluded in Section 7.

\section{PRE-PROCESSING: SLICING PLANE, DIRECTION AND THICKNESS}

Pre-processing is a fundamental set up task in slicing an object. In this step, slicing plane, slicing direction

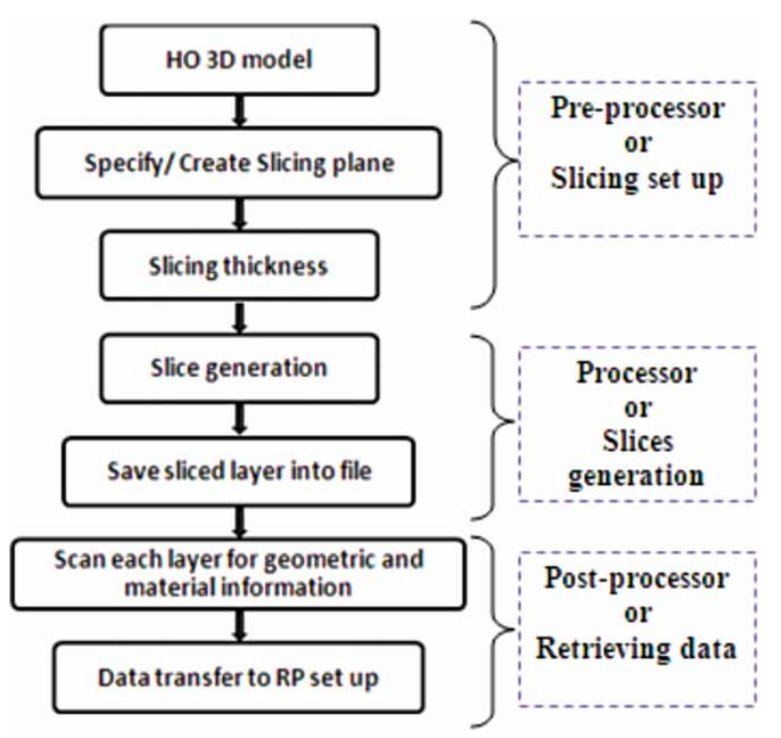

Fig. 1: Information processing for slicing of heterogeneous objects.

and slice thickness are determined to slice the 3D CAD model of homogeneous/heterogeneous object.

Any plane surface of $\mathrm{HO}$ can be selected as a slicing plane or user can also define own slicing plane i.e. the $X Y, Y Z, Z X$ plane or a specific plane defined with three points. The direction of slicing, also termed as 'Z' direction or build direction, is defined along the normal to the slicing plane.

A lot of attention is required while computing the layer thickness. A major problem in defining the thickness for rapid manufacturing is due to the stairstep effect which has lot of impact on surface quality, as shown in Fig. 2.

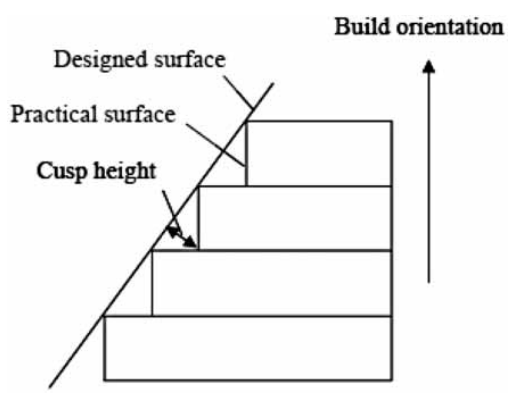

Fig. 2: Stair-step effect.

The maximum distance between manufactured surface and CAD model surface is referred to as cusp height. Dolenc et al. [2] proposed method to reduce stair-step effect through controlling the cusp height by means of adjusting the layer thickness to minimum possible. Obviously, using thinner layer thickness will increase build time if uniform slicing is employed. 
An alternative to this problem is use of adaptive slicing methods which is helpful to increase efficiency and improve the accuracy as well by varying the slice thickness. First, the CAD model is sliced uniformly into slabs with maximum layer thickness available using adaptive slicing algorithm [12]. Every slab is then re-sliced uniformly such that the cusp height is within the design tolerance as shown in Fig. 3.
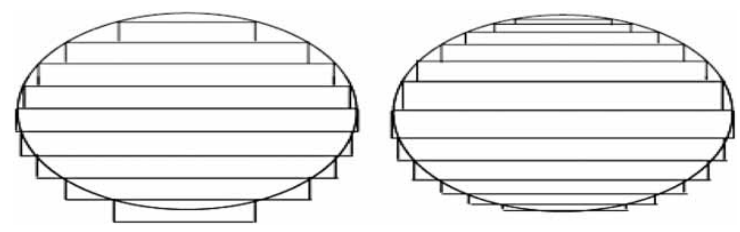

Fig. 3: Adaptive slicing [14].

A local adaptive slicing method was developed for a given group of CAD models e.g. [14]. By this method, individual models and different features of a model are independently sliced adaptively so that the accuracy is maintained and the build time is further reduced.

Mani et al. [9] proposed a region-based adaptive slicing method. The CAD model is decomposed into two regions: an adaptive layer thickness region near the curved surfaces and a common interface layer region sliced at the maximum thickness available. This method increases not only the efficiency but also provides different accuracy requirements for different regions of a model.

In the current work, an adaptive slicing algorithm is used for determining the layer thickness for slicing of heterogeneous object e.g. [15]. The layer thickness is worked out by considering the geometric as well as material distribution information. Variation in material distribution causes generation of the material stair-step effect along with the geometric stair-step effect.

The geometry-based layer thickness is computed based on the area variation rate between two adjacent slices. If the relative area variation rate between two adjacent slices is larger, the variation of the geometric features is considered to be obvious, and the layer thickness should be minimized. If the relative area variation rate between two adjacent slices is smaller, the geometric features are considered to be similar and thickness is maximized.

The material based layer thickness is computed by the gradient of material distribution and material resolution for each material. Larger is the variation in the volume fraction of each material composition, the more obvious the material staircase effect is, and so the error is larger. This maximum variation for each material is then compared with the material resolution to obtain the material-based layer thickness. The material information in each slice is stored discretely by material composition function and material resolution. Therefore, much memory space can be saved to store the material information of heterogeneous objects.

Both geometric and material based layer thicknesses are compared and minimum value is selected as slice thickness for the layer.

An example is demonstrated to find out layer thickness for a heterogeneous object as shown in Fig. 4. The object is sliced using uniform and adaptive slice method. Both methods are compared in terms of number of slices as shown in Fig. 4(b). Uniform layer thickness is taken as $0.30 \mathrm{~mm}$ and adaptive layer
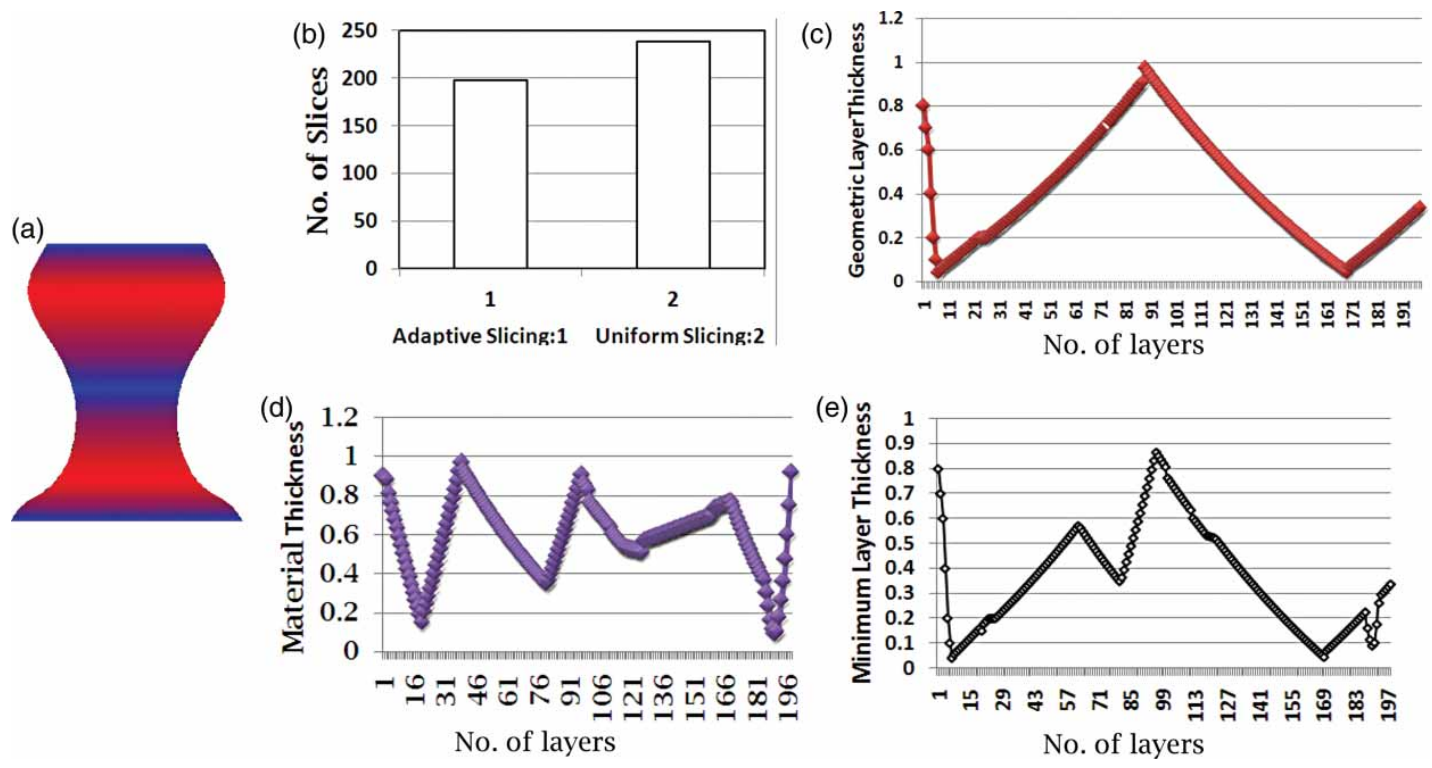

Fig. 4: Layer thickness for a heterogeneous object: (a) HO, (b) Comparison of adaptive and uniform slicing, (c) Geometric layer thickness, (d) Material thickness, and (e) Minimum layer thickness. 
thickness varies between minimum and maximum level i.e. $0.02 \mathrm{~mm}$ to $1.00 \mathrm{~mm}$ respectively. In the implemented adaptive slice method, two thicknesses for each layer are computed considering geometric and material stair-step effects as shown in Fig. 4(c) and 4(d) respectively. The minimum of two is selected for slicing the heterogeneous object as shown in Fig. 4(e).

\section{PROCESSING: SLICE GENERATION}

Pre-processing sets up some of the parameters i.e. slicing plane, slicing direction, and thickness of a layer for slice generation of heterogeneous object. Slice generation process for a heterogeneous object is completed in two steps:

- Contour generation

- Mesh generation and color mapping.

\subsection{Contour Generation}

In rapid manufacturing, the slice at a prescribed height is obtained by slicing the object with a horizontal plane at that height. The horizontal contour of the slice can be determined exactly if an accurate CAD model of the part exists. A layer is obtained by depositing material on this horizontal contour up to the next slice. However, the layer of the original CAD model between these two horizontal slices would possibly have some curved vertical surfaces as shown in Fig. 5.

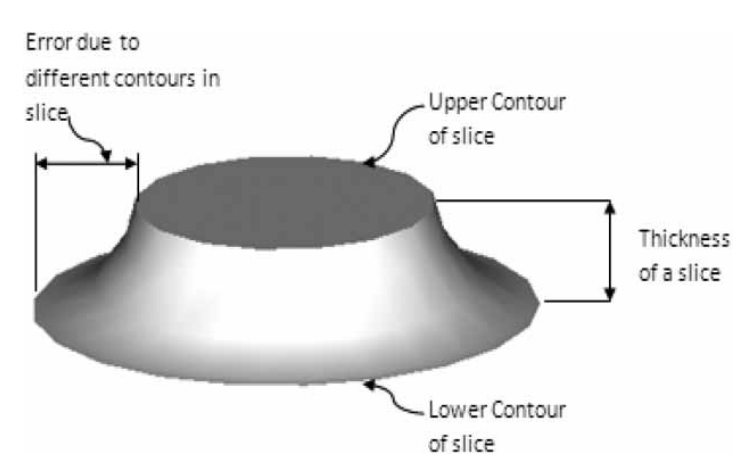

Fig. 5: Curved surface along the thickness of a slice.

Circular approximation method is implemented to approximate the contour of layers with vertical curved surfaces.

\subsection{Mesh Generation and Color Mapping}

The boundary mesh generation algorithm is extensively used in traditional solid modeler for homogeneous object modeling. Generally, the boundary mesh generation method can be divided into three phases: edge discretization, face subdivision, and triangulation. For the object to be faceted, a working face and a working edge are selected. Each working edge is first discretized into a collection of lines until the approximation error between the subdivided lines and the working edge has fallen within a given tolerance limit. A list of points on the working edge is obtained. The points derived from edge discretization stage are added to this structure and the grids on each face are initialized. The number of grid lines is determined based on surface tolerance and other input parameters e.g. normal tolerances etc. The mesh for each face is then generated through the triangulation process.

It should be particularly noted that the above boundary mesh generation is purely based on geometric criteria and the generated meshes approximate the object well in terms of geometric properties only. But such facets are not sufficient to guarantee high fidelities in material distributions and further sub-faceting process are needed.

Reference based approach solve this problem and offers functional material distribution and local control over material composition using recursive re-triangulation sub-division algorithm [3]. The algorithm is also employed for local control in a heterogeneous slice. Each triangular facet in the mesh model is sub-divided into number of sub-facets for accurate and precise material distribution in the chosen region [3]. An example of meshing and re-meshing at restricted location in the object domain is shown in Fig. 6.

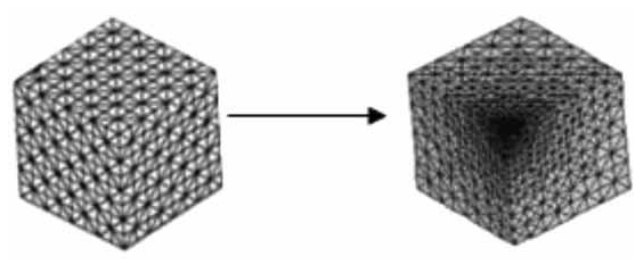

Fig. 6: Object with local control using sub-division algorithm.

\section{POST PROCESSING: RETRIEVING THE INFORMATION IN A SLICE}

Post processor deals with scanning of generated slice to store and retrieve the geometric and material information in a generated slice. An algorithm is developed to scan the layer, see Fig. 7.

The various steps of the algorithm are discussed below:

Step 1 : Number of scan lines (R) required to scan 2D slice is calculated. ' $R$ ' is calculated as the ratio of width of slice $\left(\mathrm{w}_{\mathrm{x}}\right)$ and dimension $(\mathrm{dx})$ of 


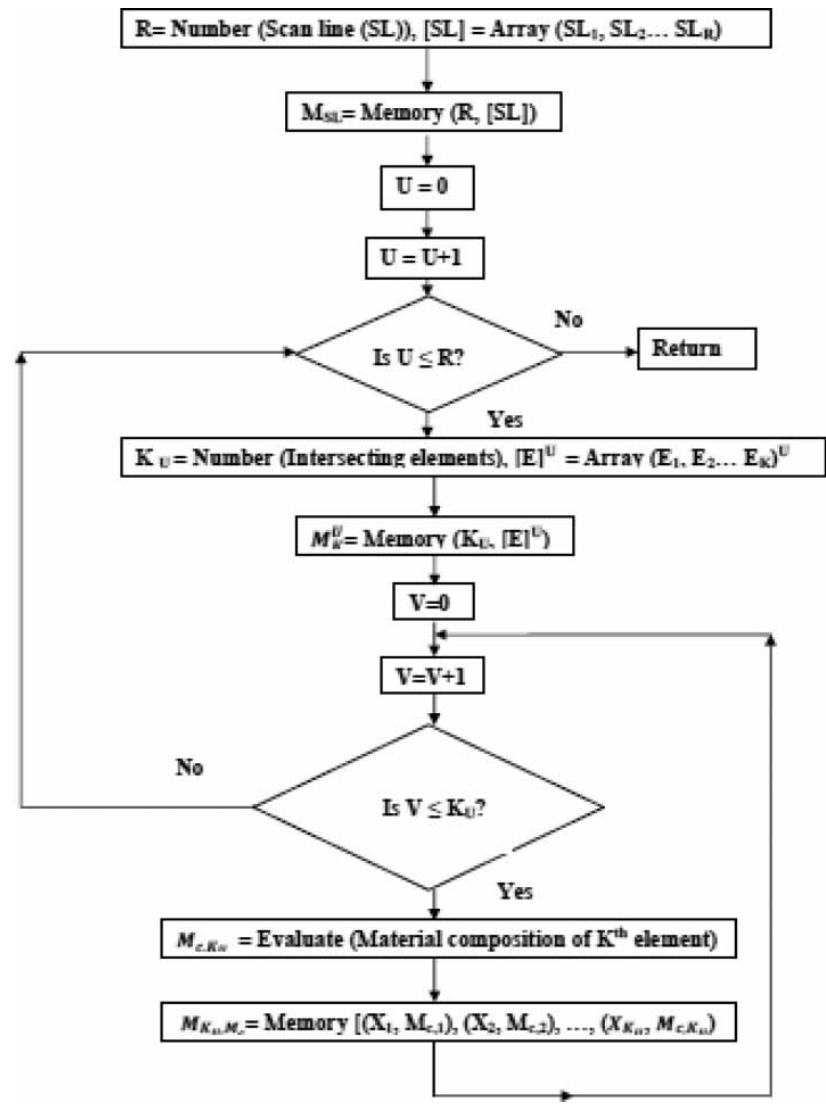

Fig. 7: Algorithm for scanning a slice.

smallest element along the scanning direction (x), given in Eqn. (4.1).

$$
\mathrm{R}=\frac{\mathrm{w}_{\mathrm{X}}}{\mathrm{dx}}
$$

Step 2 : Scan lines (SL) are identified and saved in a scan line array i.e. $[\mathrm{SL}]=\left[\mathrm{SL}_{1}, \mathrm{SL}_{2} \ldots \mathrm{SL}_{\mathrm{R}}\right]$. The information of scan lines is retrieved from the array

Step 3 : No. of scan lines and scan line array is saved in memory $\left(\mathrm{M}_{\mathrm{SL}}\right)$.

Step 4 : No. of elements $\left(\mathrm{K}_{\mathrm{U}}\right)$ intersecting scan line are identified and saved in an array $[\mathrm{E}]^{\mathrm{U}}=$ $\left[\mathrm{E}_{1}, \mathrm{E}_{2}, \ldots, \mathrm{E}_{\mathrm{K}}\right]^{\mathrm{U}}$.

Step 5 : Number of elements and element array is saved in memory $\left(\mathrm{M}_{\mathrm{K}}^{\mathrm{U}}\right)$.

Step 6 : The location $\left(\mathrm{X}_{\mathrm{K}_{\mathrm{U}}}\right)$ and material composition of each element $\left(\mathrm{M}_{\mathrm{C}, \mathrm{K}_{\mathrm{U}}}\right)$ is evaluated using geometric information and material distributed function, respectively.

A relative database of elements for each slice is created to avoid redundancy in element identification, shown in Fig. 8. The algorithm is aimed to overcome the constraints of computer memory inherent in the conventional slicing methodologies. Conventional slicing algorithm stores all facets in computer memory, which limits the size and complexity of the design and thus leads to breakdown of the rapid prototyping process. The proposed algorithm stores only the facets intersect with the cutting plane, which greatly reduces the required computer memory. The material information of facets too is the function of material composition thus can be found discretely which takes very less memory. This alleviates bottlenecks due to memory limitations and thereby facilitates rapid prototyping of large and complex models. Reduction in the required memory causes less computationally intensive searching operations in the database and reduces processing time. It results in lesser storage space and improved processing for querying geometric and material information for an element.

\section{FILE FORMATS FOR SLICE GENERATION DATA}

After the 3D object model is transferred to the process planning software, it is sliced to generate the layer information in a slice file format. The slice format must enable the storage of all the geometrical data of the layer, including the layer thickness. Also, it must have provision to store material and processrelated information, necessary for rapid manufacturing of heterogeneous objects. Some filer formats for slice generation module are discussed below:

- Stereolithography Contour (SLC) [7]: The SLC format represents individual 2D slices of a 3D object as contours representing internal and external boundaries. However, assumed specific Z-slice distance is not suitable for a crossplatform interchange format.

- Common Layer Interface (CLI) [7]: CLI, whose development started in a European BRITE/EURAM project, is aimed at providing a simple, efficient and unambiguous slice format for data input to all LM systems.

- Layer Exchange ASCII format (LEAF) [11]: The format consists of two sections; a header section and a geometry section. The header section contains preprocessing information.

The geometry section contains the layer-based geometrical data of the HO. There exists no industry standard for 2D slice data to supplement the almost universal use of STL for 3D models. As evidenced by some of the proposals described above, there is a need for such a format to provide a common basis for communicating layer information. Of the some available slice formats i.e. SLC, CLI, LEAF etc., CLI is the most complete since they provide the means for transmitting additional information beyond geometry. No non-geometric information is defined in CLI. Although development was commenced by a group of automotive companies, the current version of CLI has been enhanced through collaboration with a second BRITE/EURAM project for use with medical scan data. In our work, sliced layer information is saved in LEAF 


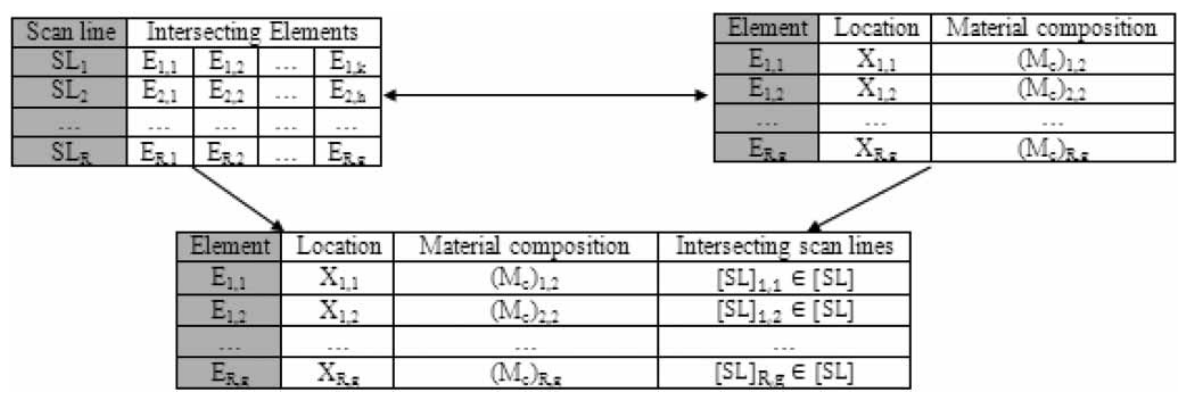

Fig. 8: Database for storing and retrieving element information.

files. The information contained in files is composed of materials information, lines, arcs and circles. This data can be easily extracted and used as control data for RP machines.

\section{EXAMPLES}

The few realistic examples are demonstrated to verify the implementation of proposed algorithms.

An example of a heterogeneous object, validating slice generation and information storage and retrieval system, is shown in Fig. 9. The object has material gradient in vertical direction. Thus, each slice has constant material composition across the cross-section.

The algorithm improves performance by reducing the time for writing and reading the data and reducing file sizes for typical large datasets. The performance statistics are summarized in Tab. 1 for the three sections shown in Fig. 9. The parameters i.e. size, processing and retrieving time are compared for conventional $(\mathrm{X})$ and proposed algorithm $(\mathrm{Y})$. The processing times refer to time used to scan the object, generate

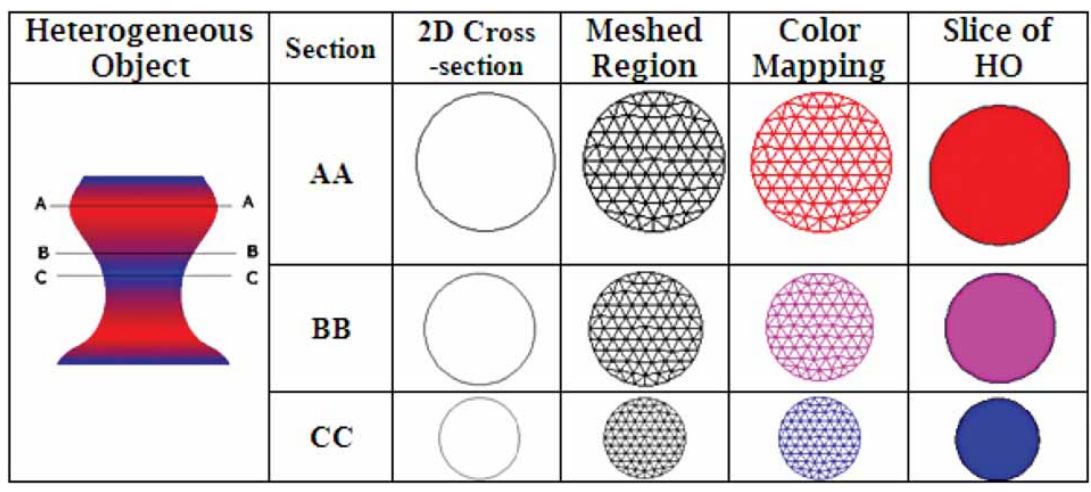

Fig. 9: Slice generation for a heterogeneous object.

\begin{tabular}{|c|c|c|c|c|c|c|}
\hline & \multicolumn{2}{|c|}{ Size (KB) } & \multicolumn{2}{|c|}{ Processing time (seconds) } & \multicolumn{2}{|c|}{ Retrieving time (seconds) } \\
\hline & $X$ & Y & $X$ & $Y$ & $X$ & Y \\
\hline Slice & 174 & 113 & 0.343 & 0.098 & 0.054 & 0.012 \\
\hline Slice at $B B$ & 132 & 98 & 0.155 & 0.052 & 0.031 & 0.009 \\
\hline Slice at CC & 96 & 76 & 0.072 & 0.013 & 0.012 & 0.002 \\
\hline
\end{tabular}

Tab. 1: Performance statistics for a heterogeneous object shown in Fig. 9.
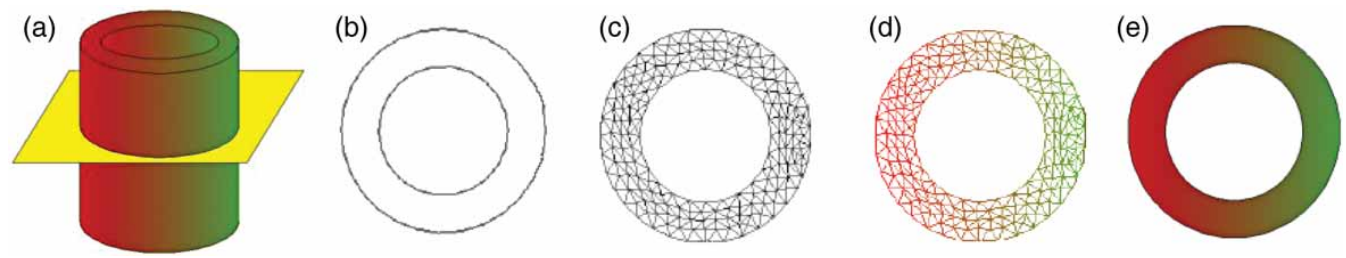

Fig. 10: Slice generation for a heterogeneous cylinder: (a) HO with Intersection Plane, (b) 2D Extracted region, (c) Meshed region, (d) Color mapping against material information, and (e) Slice of HO. 

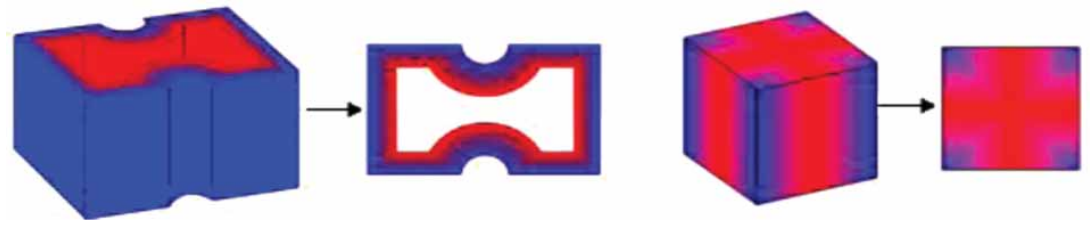

Fig. 11: Slice generation for the few heterogeneous objects.

contours, mesh, material mapping and construct an internal database for geometric and material information. The retrieve time refers to read time required for searching and extracting the information from the database.

Second example demonstrating generation of a slice for the heterogeneous cylinder is shown in Fig. 10. The material composition varies linearly along horizontal axis, thus, causes material gradient exist in each slice.

Two more examples illustrating the implementation of developed algorithm are presented in Fig. 11.

\section{CONCLUSIONS}

Issues regarding slicing of heterogeneous objects for rapid manufacturing techniques are studied. The paper is mainly focused on slice generation of a 3D $\mathrm{HO}$ and storing and retrieving geometric and material information in each slice. Challenges in getting these tasks completed are described. Adaptive thickness method is used to find out the thickness of a layer minimizing the effect of geometry and material stair-step. Contour generation method with circular approximation is implemented to reduce the error between the contours of same slice. Reference based boundary mesh generation approach is implemented for meshing 2D sliced region and assigning material composition at each point in the region. An algorithm for scanning a slice is proposed to get geometric and material distribution information in a slice and some examples are discussed to verify the results.

The adaptive thickness method implemented for computing minimum layer thickness really has potential to regulate material variation effects along the geometric one. The proposed algorithm for data storage and retrieval definitely reduce the processing and retrieving time and also avoid data redundancy. Thus, the algorithm is capable of generating high quality slice data with less required memory which can be accessed immediately for downward applications in rapid manufacturing of heterogeneous object.

\section{REFERENCES}

[1] Choi, S. H.; Kwok, K. T.: A tolerant slicing algorithm for layered manufacturing, Rapid Manufacturing Journal, 8(3), 2002, 161-179. http://dx.doi.org/10.1108/135525402 10430997

[2] Dolenc, A.; Makela, I.: Slicing procedures for layered manufacturing techniques, ComputerAided Design, 26(2), 1994, 119. http://dx.doi. org/10.1016/0010-4485(94)90032-9

[3] Gupta, V.; Kasana, K.S.; Tandon, P.: Reference based geometric modeling for heterogeneous objects, Computer Aided Design and Applications, 9(2), 2012, 155-165. http://dx.doi.org/ $10.3722 /$ cadaps

[4] Holzer, F.; Fadel, G.: Design of a 3-degrees of freedom platform for the stereolithography apparatus, Rapid Manufacturing Journal, 8(2), 2002, 100-115. http://dx.doi.org/10.1108/ 13552540210420970

[5] Hope, R. L.; Roth, R. N.; Jacobs, P. A.: 1997. Adaptive slicing with sloping layer surfaces. Rapid Manufacturing Journal, 3(3), 1997, 89-98. http://dx.doi.org/10.1108/135525497 10185662

[6] Jamieson, R.; Hacker, H.: Direct slicing of CAD models for rapid manufacturing, Rapid Manufacturing Journal, 1(2), 1995, 4-12. http://dx. doi.org/10.1108/13552549510086826

[7] Jonathan, D. H.; Hod, L.: STL 2.0: A proposal for a universal multi-material additive manufacturing file format, Solid Freeform Fabrication Symposium (SFF'09), Austin, TX, USA, 2009.

[8] Kirschman, C.; Jara-Almonte, C. C.: A parallel slicing algorithm for solid freeform fabrication processes, Proceedings of the 1992 Solid Freeform Fabrication Symposium, August 3-5, Austin, TX, 1992.

[9] Mani, K.; Kulkarni, P.; Dutta, D.: Region-based adaptive slicing, Computer-Aided Design, 31(2), 1999, 317. http://dx.doi.org/10.1016/S00104485(99)00033-0

[10] Pandey, P. M.; Venkata, N.; Dhande, R.; Dhande, S. G.: Slicing procedures in layered manufacturing: a review, Rapid Manufacturing Journal, 9(5), 2003, 274-288. http://dx.doi.org/10. 1108/13552540310502185

[11] Rock, S. J.; Wozny, M. J.: A Flexible File Format for SFF, Solid Freeform Fabrication Symposium 1991, University of Texas, Austin, 1991, $1-12$.

[12] Sabourin, E.; Houser, S. A.; Bohn, J. H.: Adaptive slicing using stepwise uniform refinement, 
Rapid Manufacturing Journal, 2(4), 1996, 20-26. http://dx.doi.org/10.1108/1355254 9610153370

[13] Tata, K.; Fadel, G.; Bagchi, A.; Aziz, N.: Efficient slicing for layered manufacturing, Rapid Manufacturing Journal, 4(4), 1998, 151-167. http://dx.doi.org/10.1108/13552549 810239003
[14] Tyberg, J.; Bohn, J. H.: Local adaptive slicing, Rapid Manufacturing Journal, 4(3), 1998, 118-127. http://dx.doi.org/10.1108/13552549 810222993

[15] Wang, S.; Zhu, y.; Chen, C. S.; Zhu, X.: Digital design for functionally graded material components rapid manufacturing, In Proceedings of GMP', 2008, 491-497. 